P50

\section{APOLIPOPROTEIN E AND LOW-DENSITY, APOLIPOPROTEIN B ASSOCIATED LIPOVIRAL PARTICLES IN CHRONIC HEPATITIS C INFECTION: EVIDENCE FOR GENOTYPE- SPECIFIC MODULATION OF LIPID PATHWAYS}

doi:10.1136/gutjn-2011-300857a.50

${ }^{1} \mathrm{~S}$ Bridge, ${ }^{1} \mathrm{D}$ Sheridan, ${ }^{1} \mathrm{D}$ Felmlee, ${ }^{2} \mathrm{M}$ Crossey, ${ }^{2} \mathrm{H}$ Thomas, ${ }^{2} \mathrm{~S}$ Taylor-Robinson, ${ }^{2} \mathrm{G}$ Toms, ${ }^{3} \mathrm{D}$ Neely, ${ }^{1} \mathrm{M}$ Bassendine. ${ }^{1}$ Newcastle University; ${ }^{2}$ Imperial College London; ${ }^{3}$ Royal Victoria Infirmary

Introduction Hepatitis $\mathrm{C}$ virus (HCV) co-opts the VLDL assembly, maturation, degradation, and secretory machinery of hepatocytes. Infectious low density particles have been termed lipoviral particles (LVP). LVPs in vivo are triglyceride (TG) rich and contain at least viral RNA, HCV core protein and the VLDL components apolipoprotein $\mathrm{B}(\mathrm{apoB})$ and apoE. ApoE is a constituent of infectious HCV particles produced in cell culture, and production of infectious particles is dramatically impaired from cells in which apoE expression has been genetically silenced.

Aim To examine the relationship between LVP and apoE in vivo. Method Fasting plasma samples were obtained from 39 chronic HCV genotype $(G)$ three patients and 51 HCV G1 patients. LVP were measured using iodixanol density gradient ultracentrifugation as recently described. ${ }^{1}$ ApoE levels were determined by an automated immunonephlometric method. Demographic data were recorded and liver biochemical tests, lipid profiles and HOMA-IR were measured in all patients.

Results The mean LVP in HCV G3 was $5.2 \log 10 \mathrm{IU} / \mathrm{ml}$ with a mean LVP ratio of 0.286 , but showed wide variation $(0.03-0.96)$. This was not significantly different to LVP variation in HCV G1 we have previously reported. ${ }^{1}$ In HCV G1 we found a strong positive correlation of LVP HCV RNA with apoE levels $(r=0.488, p=0.001)$ and also with LVP ratio $(r=0.428, p=0.001)$. In contrast in HCV G3 we found a significant negative correlation of LVP HCV RNA with apoE levels $(\mathrm{r}=-0.428, \mathrm{p}=0.013)$, suggesting different utilisation of lipoprotein pathways. We also found a negative correlation of LVP in HCV G3 with HDL cholesterol $(r=-0.468, p=0.003)$ and its structural lipoprotein apoA1 $(r=-0.479, p=0.002)$ whereas we have reported no correlation of LVP in CHCVG1 with HDL or apoA1. ${ }^{1}$ Furthermore in HCV G3 we found low TG levels compared to G1 $(1.00 \pm 0.71$ vs $1.35 \pm 0.76)$ and no correlation of LVP with TG or HOMA-IR, again contrasting to G1 infection ${ }^{1}$

Conclusion This study suggests that while serum apoE quantity is a positive determinant for LVP quantity in HCV G1 infection, it is a negative determinant in HCV G3 infection. Furthermore, LVP quantity in HCV G3 is largely based on the paucity of HDL quantity and their components, rather than the parameters associated with TRL levels as in HCV G1. These differences highlight that interaction with host lipoprotein metabolism is important for HCV infection in different genotypes, but in genotype specific ways.

\section{REFERENCE}

1. Bridge SH, et al. Insulin resistance and low-density apolipoprotein B-associated lipoviral particles in hepatitis C virus genotype 1 infection. Gut 2011:60:680-7.

\section{P51 INTERFERON-INDUCED FATIGUE IN HEPATITIS C INFECTED PATIENTS IS ASSOCIATED WITH INCREASED EXPRESSION OF INDOLEAMINE 2,3-DIOXYGENASE IN PERIPHERAL BLOOD MONONUCLEAR CELLS}

doi:10.1136/gutjnl-2011-300857a.51

${ }^{1} \mathrm{M}$ Gess, ${ }^{1} \mathrm{D}$ Forton, ${ }^{2} \mathrm{~A}$ M Myint. ${ }^{1}$ St George's Hospital and Medical School; ${ }^{2}$ Laboratory for Psychoneuroimmunology, Ludwig Maximilian University, Munich, Germany

Introduction Interferon- $\alpha$ (IFN)-based antiviral therapy for chronic hepatitis $\mathrm{C}$ virus (HCV) infection commonly induces fatigue and depression. IFN-induced symptoms may result from the activation of indoleamine 2,3-dioxygenase (IDO), the rate-limiting enzyme, regulating the breakdown of tryptophan (Trp) into L-kynurenine (Kyn), expressed in immune cells and brain. IDO activation might result in Trp and serotonin depletion and accumulation of neurotoxic Kyn metabolites. IFN increases IDO gene expression in vitro and increased serum Kyn/Trp ratios have been reported in IFNtreated patients. However, a consistent relationship between Kyn/ Trp and IFN-induced symptoms in HCV has not been shown.

Aim We sought to determine the relationship between IDO mRNA expression in PBMC, serum Kyn/Trp ratio and IFN-induced symptoms.

Method Fatigue Impact Scale (FIS) and Hospital Anxiety and Depression (HADS-D) questionnaires were completed by 19 patients at baseline and after 12 weeks treatment with PEGIFN-2a and ribavirin. Blood samples were taken from patients and 14 healthy controls. Total RNA was extracted from Ficoll separated PBMC and IDO mRNA was quantified relative to GAPDH using RT-PCR (Applied Biosystems). Expression at week 12 vs baseline was calculated as fold change using the $2^{-\Delta \Delta \mathrm{Ct}}$-method. Serum Kyn/ Trp was measured using HPLC/MS/MS.

Results IDO mRNA expression at baseline was similar in HCV patients and controls $(\Delta \mathrm{Ct}=9.76 \pm 0.39, \Delta \mathrm{Ct}=9.73 \pm 0.28, \mathrm{p}=\mathrm{NS})$. In the whole cohort, there was no change in IDO expression at week 12 vs baseline $(\Delta \mathrm{Ct}=10.0 \pm 0.23$ vs $\Delta \mathrm{Ct}=9.76 \pm 0.39, \mathrm{p}=\mathrm{NS})$, although serum Kyn/Trp increased significantly $(p<0.001)$. However, patients with IFN-induced fatigue $(\Delta$ FIS $>20 ; n=7)$ showed increased IDO mRNA expression $(\Delta \Delta \mathrm{Ct}=-0.97 \pm 0.58$; median fold change $=2.57$ ) vs those without fatigue $(\Delta \Delta \mathrm{Ct}=0.96 \pm 0.53 ; \mathrm{mfc}=0.89 ; \mathrm{p}=0.02)$. Only modest increases in depression scores were seen ( $\Delta$ HADS-D $\geq 5$ in 5 patients) but no differences in $\Delta \Delta \mathrm{Ct}$ were seen compared to those with unchanged HADS-D. Baseline IDO mRNA expression was not predictive of IFN-induced fatigue or depression. There were no associations between baseline or week $12 \mathrm{Kyn} / \mathrm{Trp}$ ratios and IFN-induced fatigue or depression.

Conclusion We show for the first time that peripheral IDO gene expression in HCV patients is similar to healthy controls and IFN-treatment leads to differential induction of IDO mRNA. IFNinduced fatigue is significantly associated with increased peripheral IDO expression but not serum Kyn/Trp. Measurement of PBMC IDO mRNA may mirror CNS changes more accurately than peripheral Trp and Kyn levels. The modest prevalence of depression in this small cohort may explain the lack of association with depression.

\section{P52 MACROPHAGE INFLAMMATORY PROTEIN-1 $\alpha / C C$ CHEMOKINE LIGAND 3 AND TUMOUR-ASSOCIATED MACROPHAGES IN HEPATITIS C VIRUS-RELATED HEPATOCELLULAR CARCINOMA: RELATION TO TUMOUR PROGRESSION AND ANGIOGENESIS}

doi:10.1136/gutjnl-2011-300857a.52

${ }^{1} \mathrm{H}$ El Aggan, ${ }^{2} \mathrm{M}$ Helmy, ${ }^{3} \mathrm{~N}$ El Deeb, ${ }^{1} \mathrm{~A}$ Zeid, ${ }^{1} \mathrm{M}$ Fawzy. ${ }^{1}$ Department of Medicine (Hepatobiliary Unit); ${ }^{2}$ Department of Clinical Pathology; ${ }^{3}$ Department of Pathology, Faculty of Medicine, University of Alexandria, Egypt

Introduction Hepatitis $\mathrm{C}$ virus (HCV) is a major risk factor for development of hepatocellular carcinoma (HCC), however, the mechanism of hepatocarcinogenesis in HCV infection is still undefined. Chemokines, which can induce the migration of leucocytes and activate inflammatory/immune responses, have recently been implicated in the regulation of tumour growth.

Aim Therefore, the aim of the present work was to study the role of macrophage inflammatory protein $-1 \alpha / C C$ chemokine ligand 3 (MIP-1 $\alpha /$ CCL3), a potent macrophage chemoattractant, in the 